\title{
PERDAS DE NITROGÊNIO POR VOLATILIZAÇÃO EM SISTEMA DE PLANTIO DIRETO EM SOLO ARENOSO
}

\author{
Ana Carolina M. Moreira, Carlos H. dos Santos, Luciana B. Fabris, Juliano C. Calonego \\ Universidade do Oeste Paulista - UNOESTE, Programa de Pós-Graduação em Agronomia, Presidente Prudente, SP. E- \\ mail: carola529@hotmail.com
}

\section{RESUMO}

O objetivo do trabalho foi avaliar as perdas do $\mathrm{N}$ pela volatilização da $\mathrm{NH}_{3}$ em um sistema de plantio direto submetido à doses de N. O experimento foi conduzido na Faculdade de Ciências Agrárias, na Universidade do Oeste Paulista - UNOESTE, em Presidente Prudente/SP, em solo classificado como Argissolo Vermelho distroférrico, seguindo o delineamento em blocos casualizados, com quatro repetições, em esquema de parcelas subdivididas, sendo as parcelas constituídas por três rotações de cultura (Milho/Tremoço/Pousio/Soja; Milho+Urochloa brizantha/Soja; Urochloa brizantha/Soja) e as subparcelas por quatro doses de N (50, 100, 150 e $200 \mathrm{~kg} \mathrm{ha}^{-1}$ ). A volatilização de $\mathrm{NH}_{3}$ não sofreu influencia significativa do sistema de rotação de culturas e das doses de $\mathrm{N}$ fornecidas ao sistema de produção. A perda da $\mathrm{NH}_{3}$ está atribuída á etapa de amonificação dos resíduos orgânicos adicionados ao solo durante o manejo das culturas e, aumentou diante da entrada dos resíduos culturais da soja.

Palavras-chave: Amônia, dose, palhada, rotação de culturas, sistemas de manejo.

\section{NITROGEN LOSSES BY VOLATILIZATION IN NO-TILLAGE SYSTEM IN SANDY SOIL}

\begin{abstract}
The objective of this work was to evaluate the losses of $\mathrm{N}$ by the volatilization of $\mathrm{NH}_{3}$ in a no tillage system submitted to $\mathrm{N}$ doses. The experiment was conducted at the Faculty of Agricultural Sciences at the University of West Paulista - UNOESTE in Presidente Prudente / SP, in soil classified as Acrisol distroferric, following the randomized block design with four replications, in a subdivided plot scheme, with the plots constituted by three crop rotations (Corn / Lupine / Fallow / Soybean, Corn + Urochloa brizantha / Soybean; Urochloa brizantha / Soybean) and the subplots by four doses of $\mathrm{N}\left(50,100,150\right.$ and $\left.200 \mathrm{~kg} \mathrm{ha}{ }^{-1}\right)$. Volatilization of $\mathrm{NH}_{3}$ was not significantly influenced by the crop rotation system and the $\mathrm{N}$ rates given to the production system. The loss of $\mathrm{NH}_{3}$ is attributed to the stage of ammonification of the organic residues added to the soil during the management of the crops, and increased before the entrance of the cultural residues of the soybean.
\end{abstract}

Keywords: ammonia, dose, straw, crop rotation, management systems.

\section{INTRODUÇÃO}

O N é elemento essencial para o crescimento das plantas, sendo necessário em grande escala para proporcionar o crescimento normal dos vegetais (MELGAR et al., 1999).

Elemento de manejo e recomendação mais complexos em virtude da multiplicidade de reações químicas e biológicas a que está sujeito, podendo ser facilmente perdido no sistema soloplanta-atmosfera por processos de volatilização, lixiviação, erosão, desnitrificação e imobilização (VARGAS, 2010). 
Dentre as perdas de $\mathrm{N}$ no solo, a volatilização da amônia $\left(\mathrm{NH}_{3}\right)$ ainda éum dos grandes problemas enfrentados pelos agricultores, uma vez que há diminuiçãoda sua disponibilidade no solo, além de causar prejuízos em outros locais ou ecossistema, e converter-se em poluentes das águas superficiais, subterrâneas e da atmosfera (CANTARELLA, 2007).Dentre os fatores de solo que podem influenciar as perdas gasosas de $\mathrm{NH}_{3}$ estão $\mathrm{o} \mathrm{pH}$, poder tampão, textura, mineralogia da fração argila, umidade, capacidade de troca de catiônica (CTC), temperatura, teor de matéria orgânica e potencial redox (COSTA et al., 2004).

O conhecimento dos processos envolvidos na incorporação e na transformação do $\mathrm{N}$ no sistema solo-planta-atmosfera é indispensável ao desenvolvimento de estratégias de manejo que aumentem o seu aproveitamento pelas culturas.

O objetivo do trabalho foi avaliar a perda de $\mathrm{N}$ pela quantificação da taxa de volatilização da $\mathrm{NH}_{3} \mathrm{em}$ sistema de semeadura diretacom rotação de culturas manejadas com doses de $\mathrm{N}$.

\section{MATERIAL E MÉTODOS}

O experimento foi conduzido na Faculdade de Ciências Agrárias, na Universidade do Oeste Paulista - UNOESTE, em Presidente Prudente/SP, em solo classificado como Argissolo Vermelho distroférrico (EMBRAPA, 2013), cuja análise granulométrica (EMBRAPA, 1997) indica os teores médios de areia $\left(\mathrm{g} \mathrm{kg}^{-1}\right)=815$, silte $\left(\mathrm{g} \mathrm{kg}^{-1}\right)=71$, argila $\left(\mathrm{g} \mathrm{kg}^{-1}\right)=114$, na camada representativa da profundidade de $0-60 \mathrm{~cm}$.

A área experimental está localizada à $430 \mathrm{~m}$ de altitude, nas coordenadas geográficas 2207' S e longitude 5127' W. O clima, de acordo com a classificação de Köppen, é do tipo Cwa, com temperatura média anual de 25 o $\mathrm{C}$ e regime pluviométrico caracterizado por dois períodos distintos, um chuvoso de outubro a março e baixo índice de chuvas entre os meses de abril e setembro (Figura 1).

Figura 1. Dados climáticos da região. Presidente Prudente, 2015.

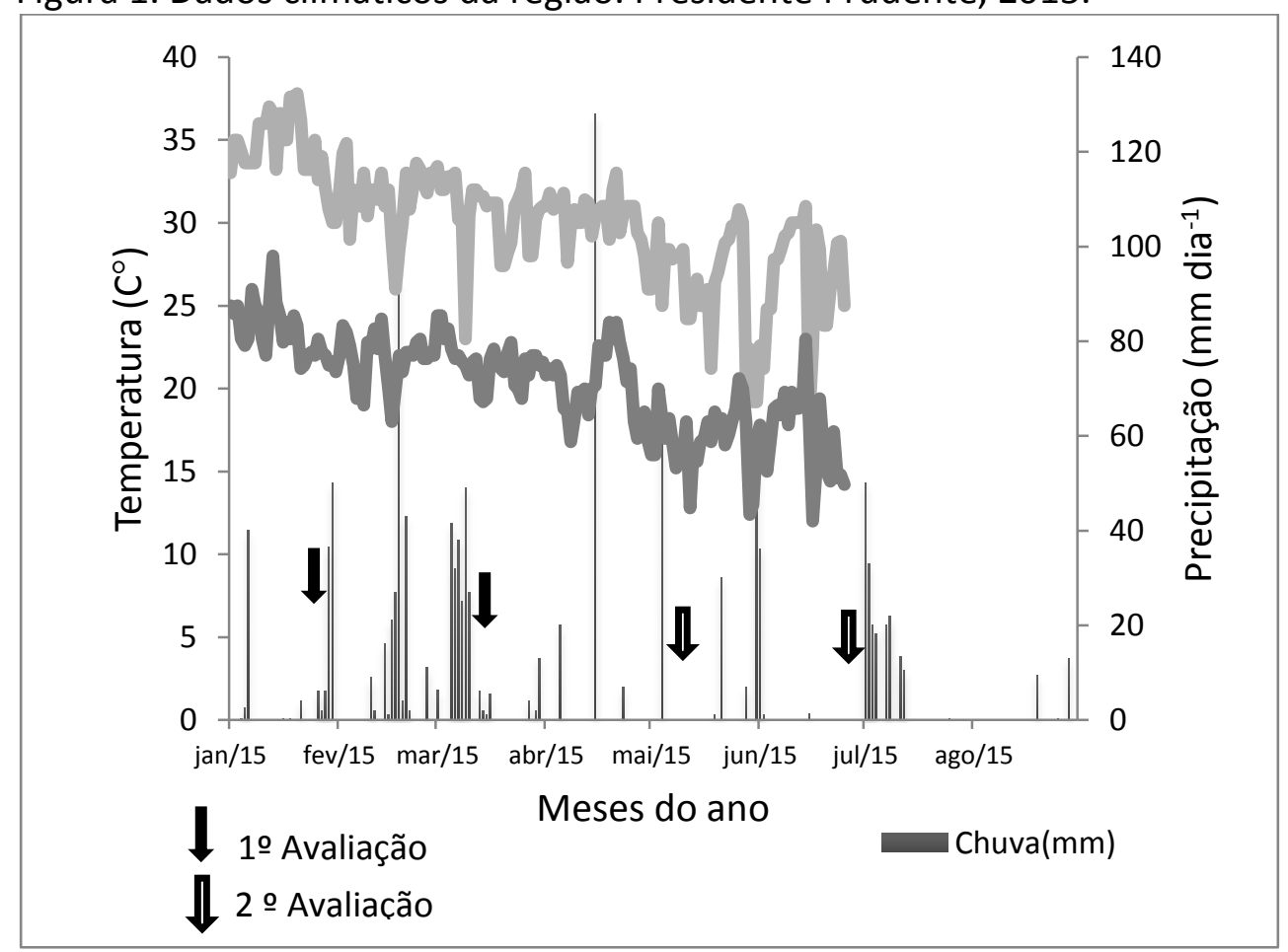

O experimento foi instalado seguindo o delineamento em blocos casualizados com quatro repetições, em esquema de parcelas subdivididas, sendo as parcelas constituídas por três rotações de culturas: 1) Milho (Mi)/Tremoço (Tr)/Pousio (Po)/Soja (So), 2) Milho+Urochloa brizantha 
(Mi+Ur)/Soja (So) e 3) Urochloa brizantha (Ur)/Soja (So) e as subparcelas por quatro doses de $\mathrm{N}$ $\left(50,100,150\right.$ e $\left.200 \mathrm{~kg} \mathrm{ha}^{-1}\right)$. As culturas principais utilizadas inseridas nas rotações foram o milho híbrido AG 5055, soja cultivar BMX Potência RR e a forrageira Urochloa brizantha (Syn. Brachiaria) cv. MG-5.

As parcelas experimentais foram definidas nas dimensões de $27 \mathrm{~m}$ (C) $\times 6,3 \mathrm{~m}$ (L), as subparcelas demarcadas com 6,0 $\mathrm{m}$ (C) $\times 6,3 \mathrm{~m}$ (L) eos blocos estavam distanciados $5,0 \mathrm{~m}$ uns dos outros.

A adubação nitrogenada, com sulfato de amônio $\left(210 \mathrm{~g} \mathrm{~kg}^{-1}\right.$ de $\left.\mathrm{N}\right)$, foi realizada durante o cultivo da cultura do milho e da forrageira nos anos de 2014 e 2015 e parcelada de acordo com cada dose, sendo $50 \mathrm{~kg} \mathrm{ha}^{-1}$ ( 2 parcelamentos de $25 \mathrm{~kg} \mathrm{ha}^{-1}$ ), $100 \mathrm{~kg} \mathrm{ha}^{-1}$ (4 parcelamentos de $25 \mathrm{~kg}$ $\mathrm{ha}^{-1}$ ), $150 \mathrm{~kg} \mathrm{ha}^{-1}$ (4 parcelamentos, sendo 2 de $25 \mathrm{~kg} \mathrm{ha}^{-1}$ e 2 de $50 \mathrm{~kg} \mathrm{ha}^{-1}$ ) e $200 \mathrm{~kg} \mathrm{ha}^{-1}$ (4 parcelamentos, sendo 2 de $25 \mathrm{~kg} \mathrm{ha}^{-1}$ e 2 de $75 \mathrm{~kg} \mathrm{ha}^{-1}$ ).

Aos 90 e 50 dias após a semeadura da soja e do milho, respectivamente, foi iniciada a quantificação da volatilização da $\mathrm{NH}_{3}$ no sistema de manejo. Em cada parcela experimental foram instalados dois coletores de $15 \times 14 \mathrm{~cm}$ (diâmetro $\times$ altura)construídoscom cilindros de PVC, cujas bases foram inseridas a $4,0 \mathrm{~cm}$ de profundidadeno solo. Cada cilindro foi protegido, na sua parte superior, com disco de polietileno com $20 \mathrm{~cm}$ de diâmetro, sendo mantida uma abertura de 2,0 $\mathrm{cm}$ entre ambos para o estabelecimento de um déficit de pressão de vapor e, assim proporcionar condições para a volatilização da $\mathrm{NH}_{3}$.

No interior do coletor de PVC foi colocado um suporte de ferro construído com um nível de altura do solo de 5,0 cm, fixado a uma Placa de Petri de vidro que abrigou em seu interior uma esponja com 2,0 × 7,5 x 7,5 cm (espessura x largura $\times$ comprimento). As esponjas foram umedecidas com $30 \mathrm{~mL}$ de ácido fosfórico $\left(0,167 \mathrm{~mol} \mathrm{~L}^{-1}\right)$, com o objetivo de captação da $\mathrm{NH}_{3}$ volatilizada do solo, e foram substituídas aos 1, 4, 8, 12, 19, 27, 34 e 43 dias após a instalação. Nas coletas, cada esponja foi recolhida, armazenada em gerbox plástico com tampa, para manter a vedação plena, e imediatamente encaminhada ao laboratório de análise química de solos/UNOESTE para extração da solução de $\left(\mathrm{NH}_{4}\right)_{3} \mathrm{PO}_{4}$ formada a partir da reação química entre a $\mathrm{NH}_{3}$ e o $\mathrm{H}_{3} \mathrm{PO}_{4}$. As esponjas foram imersas em KCl $\left(1 \mathrm{~mol} \mathrm{~L}{ }^{-1}\right)$ permanecendo em repouso por 12 horas. A lavagem das mesmas foi realizada com $500 \mathrm{mLde}$ água deionizada, em cinco lavagens sucessivas de $100 \mathrm{~mL}$ cada. Por fim, uma alíquota de $20 \mathrm{~mL}$ da solução foi destilada em destilador Kjheldal, pelo método descrito por Cantarella eTrivelin (2001).

A volatilização da $\mathrm{NH}_{3}$ foi expressa em $\mathrm{m}^{2}$ e em $\mathrm{kg} \mathrm{ha}^{-1}$ earelaçãoNH $\mathrm{N}_{3} / \mathrm{N}$-total do sistema representa a quantidade de $\mathrm{NH}_{3}$ volatilizada em cada tratamento em função do teor de $\mathrm{N}$-total do solo, também determinado pela metodologia citada por Cantarella eTrivelin (2001).

$\mathrm{O}$ cálculo para quantificação da $\mathrm{NH}_{3}$, em $\mathrm{mg} \mathrm{L}^{-1}$, está apresentado naequação a seguir: NH3 = [(Vamostr - Vbranco $) \times$ Fácido $x 14 \times 1000] / V d$,onde: Fácido: fator do ácido sulfúrico (0,0967);14: massa do N;1000: fator de conversão $\mathrm{mL}$ em L; $\mathrm{V}_{\mathrm{d}}$ : volume de extrato destilado;

A quantificação da $\mathrm{NH}_{3}$, em $\mathrm{kg} \mathrm{ha}^{-1}$, seguiu a equação: $N H 3=\left[\frac{N H 3 \times 10}{0,0177}\right] / 1000$, onde:N$\mathrm{NH}_{3}$ : teor de $\mathrm{N}$ volatilizado do solo, em mg;10: fator de conversão para hectare (ha); 0,0177: área do cilindro de PVC em $\mathrm{m}^{2}$;1000: fator de conversão $\mathrm{mg}$ em $\mathrm{kg}$.

Os resultados foram submetidos à análise de variância e os efeitos da variável qualitativa, rotação de culturas, foram comparados pelo teste Tukey ao nível de $5 \%$ de probabilidade.

\section{RESULTADOS}

$\mathrm{O}$ manejo da rotação de culturase as doses de $\mathrm{N}$ não proporcionaram efeito significativo na volatilização da $\mathrm{NH}_{3}$ durante o ciclo de desenvolvimento da soja, considerada como o primeiro período de avaliação (Tabela 2). 
Tabela 2. Volatilização da $\mathrm{NH}_{3}$ e relação $\mathrm{NH}_{3} / \mathrm{N}$-total durante o manejo da cultura da soja e do milho, submetidos a diferentes rotações de culturas e doses de $\mathrm{N}$ aplicadas. Presidente Prudente, 2015.

\begin{tabular}{|c|c|c|c|}
\hline \multirow{2}{*}{ Rotação de Culturas * } & \multicolumn{2}{|c|}{$\mathrm{NH}_{3}$} & \multirow{2}{*}{$\begin{array}{c}\mathrm{NH}_{3} / \mathrm{N} \text {-total } \\
\%\end{array}$} \\
\hline & $\mathrm{mg} \mathrm{m}^{-2}$ & $\mathrm{~kg} \mathrm{ha}^{-1}$ & \\
\hline & \multicolumn{3}{|c|}{ Manejo da soja } \\
\hline Mi/Tr/Po/So & 143,7 & 1,4 & ---- \\
\hline $\mathrm{Mi}+\mathrm{Ur} / \mathrm{Ur} / \mathrm{So}$ & 150,2 & 1,5 & ---- \\
\hline Ur/So & 152,9 & 1,5 & ---- \\
\hline \multicolumn{4}{|l|}{ Doses de $\mathrm{N}\left(\mathrm{kg} \mathrm{ha}^{-1}\right) * *$} \\
\hline 50 & 152,9 & 1,5 & 3,0 \\
\hline 100 & 138,6 & 1,4 & 1,4 \\
\hline 150 & 147,7 & 1,5 & 1,0 \\
\hline 200 & 156,5 & 1,5 & 0,8 \\
\hline Média Geral & 148,9 & 1,5 & 1,6 \\
\hline Retacãn de Culturacº & \multicolumn{3}{|c|}{ Manejo do milho } \\
\hline Mi/Tr/Po/So & 252,4 & 2,5 & ---- \\
\hline $\mathrm{Mi}+\mathrm{Ur} / \mathrm{Ur} / \mathrm{So}$ & 263,8 & 2,6 & ---- \\
\hline Ur/So & 282,6 & 2,8 & ---- \\
\hline \multicolumn{4}{|l|}{ Doses de $\mathrm{N}\left(\mathrm{kg} \mathrm{ha}^{-1}\right)^{\circ \circ}$} \\
\hline 50 & 270,0 & 2,7 & 5,4 \\
\hline 100 & 276,0 & 2,8 & 2,8 \\
\hline 150 & 266,1 & 2,7 & 1,8 \\
\hline 200 & 252,9 & 2,5 & 1,3 \\
\hline Média Geral & 266,0 & 2,7 & 2,8 \\
\hline
\end{tabular}

C.V (\%) $\mathrm{mg} \mathrm{m}^{-2}$ e kg ha ${ }^{-1} *=18,43,{ }^{* *}=16,04 ; \mathrm{dms} \mathrm{mg} \mathrm{m}^{-2} *=29,78,{ }^{* *}=26,68$, para $\mathrm{kg} \mathrm{ha}^{-1} *=0,29,{ }^{* *}=0,26 ; \mathrm{C} . \mathrm{V}(\%) \mathrm{mg}$ $\mathrm{m}^{-2}$ e kg ha ${ }^{-1 \circ}=12,44^{\circ \circ}=15,53 ; \mathrm{dms} \mathrm{mg} \mathrm{m}{ }^{-2 \circ}=35,94^{\circ \circ}=46,16$, para $\mathrm{kg} \mathrm{ha}^{-10}=0,35^{\circ \circ}=0,46$ as colunas seguidas pela mesma letra não se diferem estatisticamente entre si; foi aplicado o teste de Tukey ao nível de $5 \%$ de probabilidade.

A volatilização de $\mathrm{NH}_{3}$ ocorreu com maior intensidade no segundo período de avaliação (Tabela 2), mais especificamente durante o manejo da cultura do milho, destacando, porém, que não houve significância para os teores obtidos entre as rotações de cultura e as doses de $\mathrm{N}$.

\section{DISCUSSÃO}

A taxa de volatilização da $\mathrm{NH}_{3}$ (Tabela 2) está predominantemente associada á etapa de amonificação do $\mathrm{N}$ a partir da decomposição da palhada remanescente na superfície do solo, e que gradativamente foi incorporada naturalmente ao mesmo. SANTOS et al. (2016) também encontraram subsídios em suas pesquisas que permitem associar a perda da $\mathrm{NH}_{3}$ por volatilização com a mineralização da matéria orgânica.

O potencial de imobilização de $\mathrm{N}$ no solo também pode ter contribuído para a minimização das perdas de $\mathrm{NH}_{3}$ (Tabela 2). Em todas as rotações há a presença de, no mínimo uma gramínea, que apresenta alta relação $\mathrm{C} / \mathrm{N}$ em seu tecido. Desta forma, pode ter ocorrido uma intensificação 
da imobilização microbiana de $\mathrm{N}$ no solo devido à alta relação $\mathrm{C} / \mathrm{N}$ do material orgânico das gramíneas, redução da decomposição da matéria orgânica e da mineralização e, consequentemente, redução do teor de N passível de transformação e perda. Vitti (1998) verificou situação semelhante com a permanência significativa da palhada na superfície do solo no sistema de manejo de cana crua.

A taxa de volatilização da $\mathrm{NH}_{3}$ (Tabela 2) pode ser considerada de baixa magnitude, por exemplo, se comparada à volatilização de $\mathrm{N}$ a partir da hidrólise da ureia, a qual pode emitir até 15 $\mathrm{kg} \mathrm{ha}{ }^{-1}$ de $\mathrm{NH}_{3}$ para a atmosfera. Os resultados mostram que a volatilização de $\mathrm{NH}_{3}$ em manejos que utilizam o sulfato de amônio, em substituição à uréia, não ultrapassou o valor médio de $1,5 \mathrm{~kg}$ $\mathrm{ha}^{-1}$, independentemente da rotação de culturas ou da dose de N (Tabela 2). Lara Cabezas e Souza (2008) mencionam que o sulfato de amônio colabora na redução desta volatilização, já que o ânion sulfato reagiria com o $\mathrm{NH}_{4}{ }^{+}$formado na etapa seguinte da amonificação e da mineralização do $\mathrm{N}$, originando outro sulfato de amônio, evitando-se o aumento na concentração da $\mathrm{NH}_{3}$ livre na solução do solo esua conseqüenteperda diante do gradiente de concentração do gás em direção à atmosfera. Porém, dependendo das condições químicas do solo, os resultados podem ser contraditórios.

Considerando o teor médio geral calculado entre os manejos de cultura e as doses de $\mathrm{N}$ (Tabela 2), a volatilização de $\mathrm{NH}_{3}$ foi, em média, $78,3 \%$ superior comparado à primeira época de avaliação (Tabela 2). Esse resultadopode estar associado à adiçãodos resíduos vegetais da soja ao sistema, o qual é caracterizado por ser de rápida decomposição comparada aos resíduos das gramíneas. As plantas de cobertura, em especial as leguminosas, são importantes fontes de $\mathrm{N}$ para o sistema de produção, e, por esse motivo, o potencial de emissão de $\mathrm{N}$ a partir da degradação de seus resíduos culturais é preocupante e merece ser quantificado (JANZEN; MCGINN, 1991). Neste contexto, destaca-se a textura arenosa do solo que associada às suas características químicas e biológicas colabora para a rápida degradação da matéria orgânica e volatilização da $\mathrm{NH}_{3}$, tornando, inclusive, difícil a missão de aumentar os teores de matéria orgânica em solos arenosos (CARDIN et al., 2016). Solos arenosos tendem a apresentar humificação mais rápida da matéria orgânica, em função da sua maior macroporosidade, que promove melhores condições de aeração e maior atividade da microfauna decompositora (LUCA et al., 2008).

Os resultados apresentados na Tabela 2 são coerentes, uma vez que a adoção contínua do sistema de plantio diretoeda rotação de culturas promovemaumento da matéria orgânica do solo (BAYER et al., 2000) e da biomassa microbiana (VARGAS et al., 2005), principalmente na camada superficial. A etapa de mineralização da matéria orgânica disponibiliza até $2,0 \%$ de $\mathrm{N}$ ao ano para a cultura e, desta forma, é plausível que as perdas por volatilização sejam influenciadas pela mineralização (SANTOS et al., 2016). Fato semelhante também foi evidenciado por RAIJ (1991) em experimento cujas parcelas que não receberam adubação de $\mathrm{N}$ em cobertura apresentaramperdas consideráveis de $\mathrm{NH}_{3}$.

A relação $\mathrm{NH}_{3} / \mathrm{N}$-total indica a quantidade de $\mathrm{NH}_{3}$ volatilizada em função doteor de $\mathrm{N}$-total encontrado no perfil de solo, em cada dose de $\mathrm{N}$ aplicada (Tabela 2). À medida que houve incremento de $\mathrm{N}$ no sistema através da adubação, houve tendência de redução na taxa de volatilização. Esta observação indica que a perda de $\mathrm{NH}_{3}$ está relacionada mais especificamente com a taxa de decomposição da matéria orgânica do solo, e não com a fonte fertilizante utilizada.

Segundo Zambiazi et al. (2014), dentre os fertilizantes nitrogenados, verifica-se que a aplicação de ureia em cobertura no sistema de plantio direto, aliada às altas umidade e temperatura, proporcionam as maiores perdas de $\mathrm{N}$ por volatilização $\mathrm{NH}_{3}$. A taxa de volatilização em solos manejados comuréia é ainda maior comparada ao sulfato de amônio, podendo chegar a $50 \%$ da dose aplicada, enquanto que o encontrado neste trabalho (Tabela 2 ), considerando-se, a 
dose máxima de $200 \mathrm{~kg} \mathrm{ha}^{-1}$ de $\mathrm{N}$ aplicado às áreas manejadas com sulfato de amônio, a perda foi de 1,3\%.Lara Cabezas e Souza(2008) evidenciam também que, nos diversos sistemas agrícolas, o sulfato de amônio contribui efetivamente para atenuar as perdas gasosas de $\mathrm{NH}_{3}$, em contraste ao que ocorre com a uréia.

\section{CONCLUSÃO}

A volatilização da $\mathrm{NH}_{3}$ está atribuída à etapa de amonificação dos resíduos orgânicos adicionados ao solo durante o manejo das culturas;

A taxa de volatilização da $\mathrm{NH}_{3}$ aumentou diante da entrada dos resíduos culturais da soja.

\section{REFERÊNCIAS}

BAYER, C.; MIELNICZUK, J.; AMADO, T.J.C.; NETO MARTIN, L.; FERNANDES, S.V.Organic matter storage in a sandy clay loam Acrisol affected by tillage and cropping systems in southern Brazil. Soil and Tillage Research, v.54, n.1-2, p.101-109, 2000. https://doi.org/10.1016/S0167$\underline{1987(00) 00090-8}$

CANTARELLA, H.; TRIVELIN, P.C.O. Determinação de nitrogênio total em solos. In: RAIJ, B. et al. Análise química para avaliação da fertilidade de solos tropicais. Campinas: Instituto Agronômico de Campinas, 2001. p.262-269.

CANTARELLA, H. Nitrogênio. In: NOVAIS, R.F.; ALVAREZ, V.H.; BARROS, N.F.; FONTES, R.L.; CANTARUTTI, R.B.; NEVES, J.C.L. Fertilidade do Solo. Sociedade Brasileira de Ciência do Solo, 2007. 1017p.

CARDIN, C.A. SANTOS, C.H.; ESCARMÍNIO, M.A.Impacts of vinasse and methods of sugarcane harvesting on the availability of $\mathrm{K}$ and carbon stock of an Argisol. Revista Ceres, v.63, n.1, p.095102, 2016 https://doi.org/10.1590/0034-737X201663010013

COSTA, A.C.S.FERREIRA, J.C.; SEIDEL, E.P.; TORMENA, C.A.; PINTRO, J.C. Perdas de nitrogênio por volatilização da amônia em três solos Argilosos tratados com uréia. Acta Scientiarum Agronomy, v.26, n.4, p.467-476, 2004. https://doi.org/10.4025/actasciagron.v26i4.1809

EMBRAPA. Manual de métodos de análises de solos. 2. ed., Rio de Janeiro: CNPS, 1997. 212 p.

EMBRAPA.Sistema brasileiro de classificação de solos. 2 ed., Rio de Janeiro, CNPS, 2013. 353p.

JANZEN, H. H.; MCGINN, S. M. Volatile loss of nitrogen during decomposition of legume green manure.Soil Biology and Biology and Biochemistry, v.23, n.3, p.291-297, 1991. https://doi.org/10.1016/0038-0717(91)90066-S

LARA CABEZAS, W. A. R.; SOUZA, M. A. Volatilização de amônia, lixiviação de nitrogênio e produtividade de milho em resposta à aplicação de misturas de uréia com sulfato de amônio ou gesso agrícola. Revista Brasileira de Ciência do Solo, v.32, n.6, p.2331-2342, 2008. https://doi.org/10.1590/S0100-06832008000600012 
LUCA, E.F.; FELLER, C.; CERRI, C.C.; BARTHES, B.; CHAPLOT, V.; CAMPOS, D.C.; MANECHINI, C. Avaliação de atributos físicos e estoques de carbono e nitrogênio em solos com queima e sem queima de canavial. Revista Brasileira de Ciência do Solo, v.32, p.789-800, 2008. http://dx.doi.org/10,1590/S0100-06832008000200033

MELGAR, R.; CAMOZZI, M.E.; FIGUEROA, M.M. Guia de fertilizantes em miendas y produtos nutricionales. Buenos Aires: Instituto Nacional de Tecnologia Agropecuária, 1999. v.1, 260p.

RAIJ, B V. Fertilidade do solo e adubação. Piracicaba: Pontafós, 1991. 343 p

SANTOS, S.M.C.; ANTONANGELO, J.A.; DEUS, A.C.F.; FERNANDES, D.M. Perdas de amônia por volatilização em resposta a adubação nitrogenada do feijoeiro. Revista de Agricultura Neotropical, v.3, n.1, p.16-20, 2016.

VARGAS, V.P. Manejo da adubação nitrogenada na recuperação de estresses em milho. 2010.145f. Dissertação (Mestrado em Ciência do Solo) - Centro de Ciências Agroveterinárias, Universidade do Estado de Santa Catarina.

VARGAS, L.K.; SELBACH, P.A.; SÁ, E.S.Imobilização de nitrogênio em solo cultivado com milho em sucessão à aveia preta nos sistemas plantio direto e convencional. Ciência Rural, v.35, n.1, p.7683, 2005. https://doi.org/10.1590/S0103-84782005000100012

VITTI, A.C. Utilização pela cana-de-açúcar (cana planta) do nitrogênio da uréia (15N) e do mineralizado no solo em sistemas de manejo com e sem a queima. 1998. 93f. Dissertação (Mestrado em Ciência) - Centro de Energia Nuclear na Agricultura (CENA), Universidade de São Paulo.

ZAMBIAZI, M.A.et al. Perdas de nitrogênio por volatilização de amônia através da aplicação de ureia em solos agrícolas. $1^{\circ}$ Simpósio de Agronomia e Tecnologia em Alimentos, 2014. 